\title{
MicroRNA-153 attenuates hypoxia-induced excessive proliferation and migration of pulmonary arterial smooth muscle cells by targeting ROCK1 and NFATc3
}

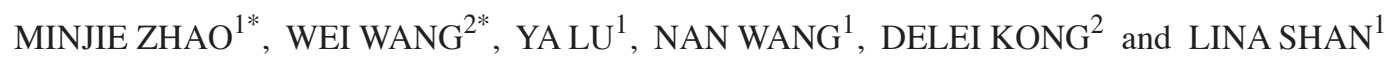 \\ ${ }^{1}$ Department of Respiratory Disease, The First Affiliated Hospital of Jinzhou Medical University, \\ Jinzhou, Liaoning 121001; ${ }^{2}$ Department of Respiratory Disease, The First Affiliated Hospital of \\ China Medical University, Shenyang, Liaoning 110000, P.R. China
}

Received May 1, 2020; Accepted December 1, 2020

DOI: $10.3892 / \mathrm{mmr} .2021 .11833$

\begin{abstract}
The aim of the present study was to explore the effect of microRNA (miR)-153 on the proliferation and migration of pulmonary artery smooth muscle cells (PASMCs) in a hypoxic condition by targeting $\mathrm{Q}$-associated, coiled-coil-containing protein kinase 1 (ROCK1) and nuclear factor of activated T cells cytoplasmic 3 (NFATc 3 ). The right ventricular systolic pressure, right ventricular hypertrophy index, medial wall thickness and medial wall area were studied at different time-points after rats were exposed to hypoxia. Western blot analysis was used to detect ROCK1 and NFATc3 protein levels. In addition, reverse transcription-quantitative (RT-q) PCR was performed to confirm the mRNA levels of miR-153, ROCK1 and NFATc3 in human (H)PASMCs under hypoxic conditions. Transfected cells were then used to evaluate the effect of miR-153 on cell proliferation and migration abilities. The association between miR-153 and ROCK1 or NFATc3 was identified through double luciferase assays. Hypoxia induced pulmonary vascular remodeling and pulmonary arterial hypertension, which resulted from the abnormal proliferation of HPASMCs. ROCK1 and NFATc3 were the target genes of miR-153 and miR-153 mimic inhibited the protein expressions of ROCK 1 and NFATc3 in HPASMCs and further inhibited cell proliferation and migration under hypoxic conditions. By contrast, the miR-153 inhibitor promoted the proliferation and migration of HPASMCs. miR-153 regulated the proliferation
\end{abstract}

Correspondence to: Dr Lina Shan, Department of Respiratory Disease, The First Affiliated Hospital of Jinzhou Medical University, 2 Beijing Road, Guta, Jinzhou, Liaoning 121001, P.R. China E-mail: shanlina321@163.com

*Contributed equally

Key words: pulmonary arterial hypertension, miR-153, Q-associated, coiled-coil-containing protein kinase 1, nuclear factor of activated $\mathrm{T}$ cells cytoplasmic 3 , hypoxia and migration of HPASMCs under hypoxia by targeting ROCK1 and NFATc3.

\section{Introduction}

Pulmonary arterial hypertension (PAH) is a severe pathophysiological syndrome characterized by pulmonary vasoconstriction and structural remodeling of blood vessel walls, which in turn lead to increased vascular resistance and right ventricular dysfunction $(1,2)$. The causes of PAH are complex, with hypoxia being one of the most important factors $(3,4)$. As the main cells in pulmonary arteries, pulmonary artery smooth muscle cells are sensitive to hypoxia (5). Under hypoxic conditions, abnormal proliferation and migration of pulmonary artery smooth muscle cells (PASMCs) cause pulmonary stenosis or occlusion and further induce the occurrence and development of PAH (6). Therefore, strategies to inhibit the abnormal proliferation and migration of PASMCs and reverse pulmonary vascular remodeling are particularly important in the treatment of PAH.

MicroRNAs (miRNAs/miRs) are small, endogenous RNAs 22 nucleotides in length (7). miRNAs serve an important regulatory role in organism development, cell differentiation, proliferation and apoptosis, as well as in pathological and physiological activities, including tumorigenesis (8-10). miRNAs commonly negatively regulate the expression of target genes by specifically binding to the 3'-untranslated region (3'-UTR) of the target mRNA molecule (11). Recently, it has been reported that a number of miRNAs serve a vital role in the occurrence and development of $\mathrm{PAH}$, highlighting their potential use as a biomarker and therapeutic tool for PAH $(12,13)$. In addition, miRNAs regulate the PASMC phenotype and pulmonary vascular remodeling under hypoxia (14). For example, decreased expression of miR-34a in PASMCs induces cell proliferation, increases the expression of platelet growth factor receptor $\alpha$ and promotes the development of PAH; this phenomenon is reversed by overexpression of miR-34a (15). miR-143-5p modulates pulmonary artery smooth muscle cell functions in hypoxic pulmonary hypertension through targeting hypoxia-inducible factor-1 (HIF-1) $\alpha$ (16). miR-153 is involved in different pathophysiological processes in human 
diseases, such as suppressing lung cancer $(17,18)$, inhibiting pulmonary fibrosis (19) and inhibiting angiogenesis under hypoxic conditions (20). However, the effect of miR-153 on PAH has yet to be reported.

Q-associated, coiled-coil-containing protein kinase 1 (ROCK1) and nuclear factor of activated T cells cytoplasmic 3 (NFATc3) serve an important role in the formation of hypoxic pulmonary hypertension. Inhibitors of these molecules significantly inhibit the proliferation and migration of pulmonary smooth muscle cells, constraining the development of pulmonary hypertension (21-23). miRNAs are used to target NFATc 3 to inhibit PAH induced by the proliferation of human (H)PASMCs (24). The present study explored the ability of miR-153 to inhibit pulmonary vascular remodeling induced by hypoxia by targeting ROCK1 and NFATc3, providing new ideas for treatment of PAH.

\section{Materials and methods}

Animal experiment. Healthy male Sprague-Dawley (SD) rats (6-8 week-old; 180-200 g; $n=30$ ) were purchased from the Experimental Animal Center of Jinzhou Medical University (Jinzhou, China). The present study adhered to the Guide for the Care and Use of Laboratory Animals published by the US National Institutes of Health (NIH Publication No. 85-23, revised 1996) (25) and all experimental protocols were approved by the Animal Experimentation Ethics Committee of Jinzhou Medical University (approval no. 2019065). The rats were randomly divided into five groups including one control group (0 day) and four hypoxia groups (1, 2, 4 and 6 weeks). Subsequentially, six rats in each group were sacrificed for investigation at every time-point (0 day, 1, 2, 4 and 6 weeks). All rats in the normal and control groups survived until sacrifice. The hypoxic rats were housed in a gas chamber with a gas control delivery system (Oxycycler model A84XOV; BioSpherix, Ltd.) for $12 \mathrm{~h}$ per day. The duration of the experiments was $\sim 6$ weeks (including 0 day, 1, 2, 4 and 6 weeks). During exposure to hypoxia, oxygen concentration was set at $10 \pm 0.5 \%$ and age-matched male rats were housed under normoxic conditions (21\% oxygen) as normal controls. All animals received humane care during the study. All rats were housed at $18-23^{\circ} \mathrm{C}$ with $40-60 \%$ humidity, $12 \mathrm{~h}$ light/dark cycles, and free access to food and water. Animal health and behavior were monitored once a week.

Measurement of the right ventricular systolic pressure (RVSP) and right ventricular hypertrophy index (RVHI) in rats. The rats were anesthetized by the intraperitoneal injection of $10 \%$ chloral hydrate $(400 \mathrm{mg} / \mathrm{kg})$ and fixed on an experimental operating table. No signs of peritonitis were observed after the administration of $10 \%$ chloral hydrate. The right external jugular vein was exposed and dissociated. After the vessel was cut, a prefilled copper heparin PE-50 catheter was quickly inserted into the right ventricle via the vessel along the incision. The other end of the catheter was connected to a multi-lead physiological recording instrument by a pressure sensor. The pulmonary artery systolic pressure was indirectly reflected by recording the RVSP. Subsequently, rats were sacrificed directly by cervical dislocation while unconsciousness.
After cessation of breathing and heartbeat, lung and heart samples were separated. The right ventricular (RV) free wall was cut along the interventricular septum and the left ventricle and ventricular septum $(\mathrm{LV}+\mathrm{S})$ were separated. Finally, the RVHI was calculated after weighing based on the following formula: $\mathrm{RVHI}=\mathrm{RV} /(\mathrm{LV}+\mathrm{S})$.

Morphological investigation. Lung tissues were obtained from anesthetized rats and sagittal sections of right lungs were fixed in $4 \%$ paraformaldehyde solution for $72 \mathrm{~h}$, embedded in paraffin and serially sectioned at $4 \mu \mathrm{m}$. Following dehydration using alcohol dehydration (70, 80, 90, 100 and 100\% alcohol; 30 min per step) and clearing with benzene, sections were deparaffinized as follows: Benzene for $5 \mathrm{~min}$; $100 \%$ alcohol for $5 \mathrm{~min}$; $90 \%$ alcohol for $2 \mathrm{~min}$; $80 \%$ alcohol for $2 \mathrm{~min}$; $70 \%$ alcohol for $2 \mathrm{~min}$; and $\mathrm{dd}_{2} \mathrm{O}$ for $2 \mathrm{~min}$. Tissue sections were then stained with hematoxylin for $10 \mathrm{~min}$ at room temperature, washed with water for $15 \mathrm{~min}$ and then stained with eosin for $1 \mathrm{~min}$ at room temperature. Pulmonary arteries $(\mathrm{n}=5$; external diameters 50-100 $\mu \mathrm{m}$ ) were randomly chosen from each rat and observed using an optical light microscope (magnification, x200). The images were shot and analyzed with Image-Pro Plus, Version 6.0 (Media Cybernetics, Inc.). The degree of pulmonary artery remodeling was assessed by calculating the percentage of the medial wall thickness (WT\%) and medial wall area (WA\%) according to the following equations: WT $\%=2 \times$ WT/external diameter) $\mathrm{x} 100$; $\mathrm{WA} \%=($ medial WA/total vessel area $) \times 100$. The pulmonary arteries were isolated from the remaining lung tissues and further used for western blot analyses.

Cell experiments. HPASMCs (ScienCell Research Laboratories, Inc.) were cultured in a smooth muscle cell culture medium with fetal bovine serum (FBS; Lonza Group, Ltd.) In the normal group, the cells were cultured at $37^{\circ} \mathrm{C}$ under the condition of $21 \% \mathrm{O}_{2}, 5 \% \mathrm{CO}_{2}$ and $74 \% \mathrm{~N}_{2}$. In the hypoxia group, the cells were cultured at $37^{\circ} \mathrm{C}$ under the condition of $3 \% \mathrm{O}_{2}, 5 \% \mathrm{CO}_{2}$ and $92 \% \mathrm{~N}_{2}$. After exposure to hypoxia for 6,12 and $24 \mathrm{~h}$, the mRNA expression levels of miR-153, ROCK1 and NFATc3 in HPASMCs were evaluated by reverse transcription-quantitative (RT-q)PCR. Transfection efficiency was estimated by evaluation of green fluorescent protein expression under a fluorescence microscope.

Cell transfection. $\mathrm{miR}-153$ (miR-153 mimic), negative control miRNA (mimic control), miR-153 inhibitor (miR-153 inhibitor) and negative control (inhibitor control) were purchased from Shanghai GenePharma Co., Ltd. The sequences of the transfection vectors were as follows: miR-153 mimics, 5'-UUGCAUAGUCACAAAAGUGAUC-3'; mimic control, 5'-UUCUCCGAACGUGUCACGU-3'; miR-153 inhibitor, 5'-GAUCACUUUUGUGACUAUGCAA-3'; inhibitor control, 5'-CAGUACUUUUGUGUAGUACAA-3'. Subsequently, $100 \mathrm{nM}$ miR-153 mimic, mimic control, miR-153 inhibitor or inhibitor control were transfected into HPASMCs $\left(1 \times 10^{6}\right.$ cells/well) using the GP-siRNA-Mate kit (Shanghai GenePharma Co., Ltd.) according to the manufacturer's instructions. The transfection controls were non-targeting controls. At $48 \mathrm{~h}$ post-transfection, cells were used for subsequent experiments. 
Table I. Primer sequences for reverse transcription-quantitative PCR.

\begin{tabular}{|c|c|c|}
\hline Gene & Primer sequence $\left(5^{\prime} \rightarrow 3^{\prime}\right)$ & Length of product (bp) \\
\hline \multicolumn{3}{|c|}{$\operatorname{miR}-153$} \\
\hline & F: AGCCGCTTGCATAGTCACA & 78 \\
\hline & R: AGAGCAGGGTCCGAGGAT & 78 \\
\hline \multicolumn{3}{|c|}{ 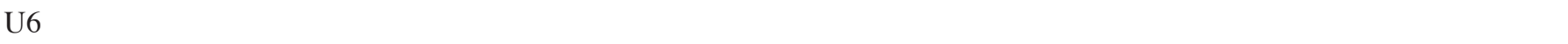 } \\
\hline & F: CGCTTCGGCAGCACATATAC & 81 \\
\hline & R: TTCACGAATTTGCGTGTCATC & 81 \\
\hline \multicolumn{3}{|c|}{ ROCK1 } \\
\hline & F: CTGCAACTGGAACTCAACCAAGAA & 167 \\
\hline & R: TTAGCACGCAATTGCTCAATATCAC & 167 \\
\hline \multicolumn{3}{|c|}{ NFATC3 } \\
\hline & F: TCCACСТССАТСТАСТТТААССА & 162 \\
\hline & R: TTGGGACCACCTAATGGGCT & 162 \\
\hline \multicolumn{3}{|c|}{ GAPDH } \\
\hline & F: CATGAGAAGTATGACAACAGCCT & 113 \\
\hline & R: AGTCCTTCCACGATACCAAAGT & 113 \\
\hline
\end{tabular}

RNA isolation and RT-qPCR. Total RNA was isolated from HPASMCs using a TRIzol ${ }^{\circledR}$ reagent kit according to the manufacturer's protocol (Thermo Fisher Scientific, Inc.). RNA extraction, cDNA synthesis (total $20 \mu \mathrm{l}$ ) and quantitative analysis (total $20 \mu \mathrm{l}$ ) was performed using the Hairpin-it miRNA qPCR quantification kit (Shanghai GenePharma Co., Ltd.) according to the manufacturer's protocol. U6 was used as an internal reference. The reverse transcription procedure for U6 small nuclear RNA and miRNA- 153 was: $25^{\circ} \mathrm{C}$ for $30 \mathrm{~min}$, $42^{\circ} \mathrm{C}$ for $30 \mathrm{~min}, 85^{\circ} \mathrm{C}$ for $5 \mathrm{~min}$ and storage at $4^{\circ} \mathrm{C}$. The reverse transcription procedure for GAPDH, ROCK1 and NFATc3 was: $42^{\circ} \mathrm{C}$ for $45 \mathrm{~min}, 85^{\circ} \mathrm{C}$ for $5 \mathrm{~min}$ and storage at $4^{\circ} \mathrm{C}$. The PCR reaction program was as follows: Pre denaturation at $95^{\circ} \mathrm{C}$ for $5 \mathrm{~min}$, followed by 40 cycles of $95^{\circ} \mathrm{C}$ denaturation for $12 \mathrm{sec}$ and annealing at $62^{\circ} \mathrm{C}$ for $40 \mathrm{sec}$. The primer sequences are listed in Table I. miRNA levels was quantified using the $2^{-\Delta \Delta C q}$ method (26) and expression value was normalized to the internal control gene U6. The assay was performed in triplicate.

Cell proliferation assay. Cell proliferation was assessed using a cell counting kit (CCK-8; Dojindo Molecular Technologies, Inc.). Transfected HPASMCs were seeded in a 96-well plate at $5 \times 10^{3}$ cells/well. After the cells were incubated for $12 \mathrm{~h}$ under hypoxia, $10 \mu \mathrm{l}$ of CCK-8 was added to each well and the cells were incubated at $37^{\circ} \mathrm{C}$ for $2 \mathrm{~h}$. The absorbance (optical density value) was measured at $450 \mathrm{~nm}$.

Wound healing (cell migration) assay. Cell migration ability was measured by a wound healing assay. In brief, miR-153 mimic, mimic control, miR-153 inhibitor and inhibitor control were transfected into HPASMCs. Cells, which were cultured in medium containing $2 \%$ FBS, were plated in 24-well cell culture plates at a density of $4 \times 10^{5}$ cells/well and cultured to confluence. Subsequently, a $200 \mu 1$ sterile pipette tip was used to scratch a straight line in the monolayer of cells. After washing three times with PBS, the remaining cells were cultured in serum-free medium for another $12 \mathrm{~h}$. Images were captured at 0 and $12 \mathrm{~h}$ using an inverted light microscope (IX71; Olympus Corporation; magnification, x100). The wounded area was manually outlined based on images acquired immediately after wounding and $12 \mathrm{~h}$ later. Only wounds with areas between 1.2 and $2.5 \mathrm{~mm}^{2}$ were analyzed to ensure that data would be comparable. Finally, four fields of view were observed for each sample. The experiments were repeated three times.

Transwell cell migration assay. Cell migration was evaluated using $8-\mu \mathrm{m}$ pore Transwell chambers (Corning Life Sciences). The cells were suspended in a serum-free medium at a density of $1 \times 10^{5}$ cells $/ \mathrm{ml}$. Then, $200 \mu \mathrm{l}$ cell suspension was added into the upper chamber and $600 \mu \mathrm{l}$ medium containing $5 \%$ FBS was added into the lower chambers. After $12 \mathrm{~h}$ incubation at $37^{\circ} \mathrm{C}$ under hypoxic conditions, the cells had migrated to the lower chamber. Non-migratory cells were removed using a cotton swab. Cells were fixed with $95 \%$ ethanol for $15 \mathrm{~min}$ at room temperature and washed three times with PBS (5 min per wash). Subsequently, cells were stained with $0.1 \%$ crystal violet (Sigma-Aldrich; Merck $\mathrm{KGaA}$ ) for $10 \mathrm{~min}$ at room temperature. Following washing with tap water, migratory cells were observed using an inverted light microscope (magnification, $\mathrm{x} 200$ ).

Western blot analysis. ProteinExt Mammalian Total Protein Extraction kit (TransGen Biotech Co.,Ltd.) was used to isolate total proteins. Protein concentrations were determined using a Pierce bicinchoninic acid protein assay kit (Pierce; Thermo Fisher Scientific, Inc.) and equal amounts of the protein $(\sim 50 \mu \mathrm{g})$ were isolated by $10 \%$ SDS-PAGE and transferred onto polyvinylidene fluoride membranes. Membranes were 
A

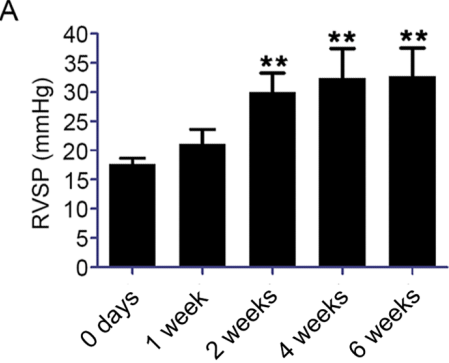

C

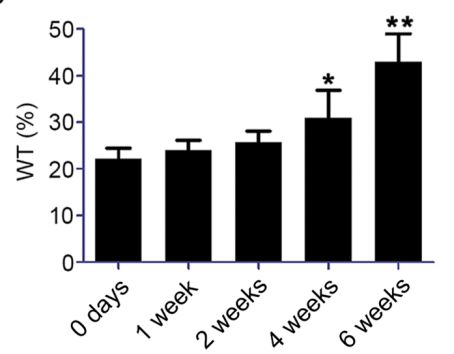

B

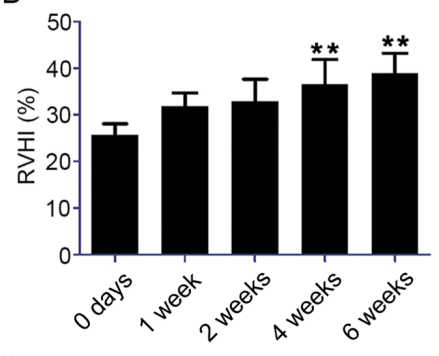

D

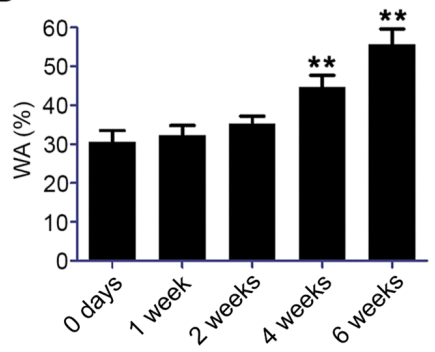

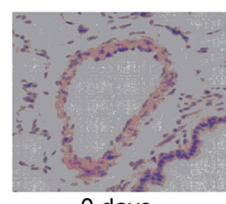

0 days

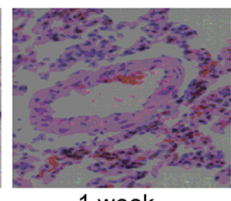

1 week

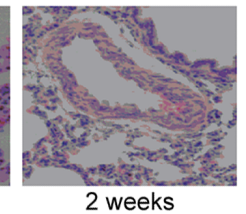

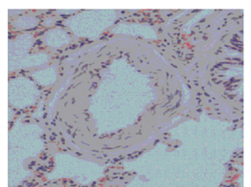

4 weeks

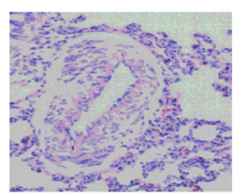

6 weeks

Figure 1. Establishment of a rat model of HPH and identification of rat pulmonary artery smooth muscle cells. (A) RVSP and (B) RVHI, calculated as the weight ratio between the RV and LV plus S: RV/LV+S; (C) WT and (D) WA of pulmonary arteries of 50-100 $\mu \mathrm{m}$ in diameter. (E) Hematoxylin and eosin staining of the smooth muscle layer of pulmonary vessels in the hypoxic condition. Scale bar, $100 \mu \mathrm{m}$. Data represent the mean \pm standard deviation, $\mathrm{n}=3$. Comparisons were performed using one-way ANOVA followed by Dunnett's post-hoc test. ${ }^{*} \mathrm{P}<0.05$ and ${ }^{* *} \mathrm{P}<0.01$ vs. control at day 0 . HPH, hypoxia-induced pulmonary hypertension; RVSP, right ventricular systolic pressure; RVHI, right ventricular hypertrophy index; RV, right ventricle; LV, left ventricle; S, septum; WT, medial wall thickness; WA, medial wall area.

then blocked for $1 \mathrm{~h}$ at room temperature with $5 \% \mathrm{BSA}$ in TBST $(0.1 \%$ Tween-20). The membranes were then incubated overnight at $4^{\circ} \mathrm{C}$ with primary antibodies for detection of the following: ROCK1 (cat. no. ab134181; 1:1,000; Abcam), PCNA (cat. no. K200030M; 1:1,000; Beijing Solarbio Science \& Technology Co., Ltd.), matrix metalloproteinase (MMP)-2 (cat. no. A00286; 1:300; Wuhan Boster Biological Technology, Ltd.), NFATc3 (cat. no. sc-8405; 1:200; Santa Cruz Biotechnology, Inc.) and $\beta$-actin (cat. no. ab8227; 1:1,000; Abcam). The membranes were washed three times and were then incubated for $1 \mathrm{~h}$ with horseradish peroxidase secondary detection antibody (cat. nos. A0208 and A0216; 1:10,000; Beyotime Institute of Biotechnology); $\beta$-actin was used as internal controls. The signals of bands were measured using enhanced chemiluminescence (ECL) detection kit (Beyotime Institute of Biotechnology) and visualized on a commercial X-ray film. Relative protein levels were semi-quantified using Image J software (version 1.41; National Institutes of Health).

Immunofluorescence experiments. The transfected HPASMCs were seeded on cover slips in a 24 -well plate at a density of $4 \times 10^{4}$ cells $/ \mathrm{ml}$. After $48 \mathrm{~h}$, the cells were fixed with 4\% paraformaldehyde for $30 \mathrm{~min}$ and washed three times with phosphate-buffered saline. Triton X-100 (1\%; $200 \mu \mathrm{l}$; Sigma-Aldrich; Merck KGaA) was added into each well for $30 \mathrm{~min}$ at room temperature. After the samples were washed three times with PBS, HPASMCs were blocked with goat serum (1:500; Beijing Solarbio Science \& Technology Co., Ltd.) at room temperature for $30 \mathrm{~min}$. The cells were then incubated overnight at $4^{\circ} \mathrm{C}$ with the primary antibody for detection of NFATc3 (cat. no. sc-8405; 1:200; Santa Cruz Biotechnology, Inc.). The cells were then incubated for $2 \mathrm{~h}$ at room temperature with an Alexa Fluor 647 fluorescently labeled anti-mouse secondary antibody (cat. no. A0473; 1:100; Beyotime Institute of Biotechnology). Cells were finally double-stained with DAPI (1:1,000, Invitrogen; Thermo Fisher Scientific, Inc.) for $5 \mathrm{~min}$ at room temperature and then viewed by fluorescence microscopy.

Dual-luciferase reporter assay. Luciferase reporter assays were performed to verify that miR-153 directly interacted with the 3'-UTR of ROCK1 and NFATc3. The target association between miR-153 and ROCK1 or NFATc3 was verified by the TargetScan (www.targetscan.org/vert_72; version 7.0) database (27). The wild-type (WT) miR-153-ROCK1 or miR-153-NFATc3 (WT ROCK1 or WT NFATc3) and mutant miR-153-ROCK1 or miR-153-NFATc3 (Mut ROCK1 or Mut NFATc3) plasmids were designed and constructed according to the database of TargetScan and miRanda (www.mirbase. org). The 3'-UTR of 3'-UTR/NFATc3 or ROCK1 containing the complementary binding site of miR-153 was directly synthesized by Hanbio Biotechnology Co., Ltd. into the psiCHECK-2 vector (Promega Corporation) to construct the ROCK1 or NFATc3 WT luciferase reporter vector (ROCK1-WT or NFATc3-WT). To establish the ROCK1 mutant luciferase reporter vector (ROCK1-MUT), the site-specific mutagenesis system (Invitrogen; Thermo 
A

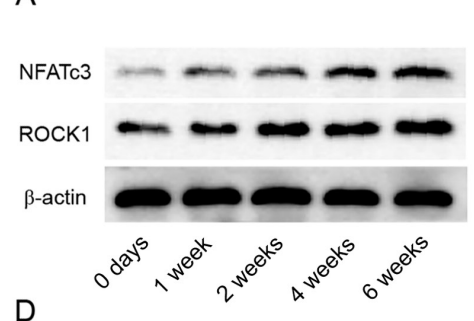

D

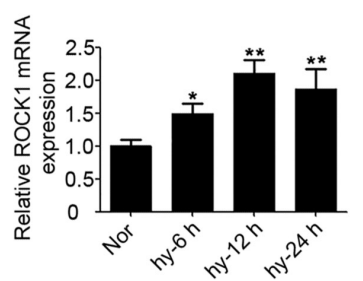

B

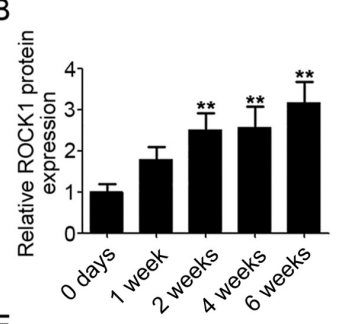

E

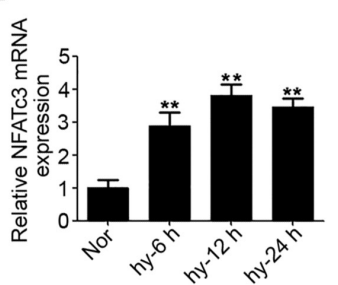

C

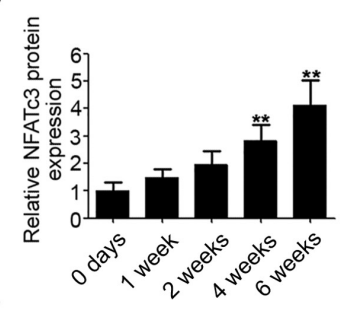

F

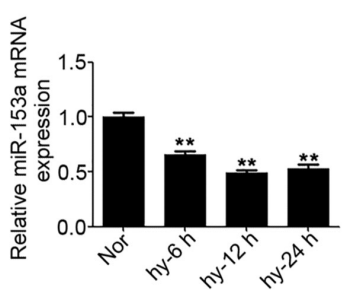

Figure 2. Protein expression levels of ROCK1 and NFATc3 in HPASMCs. (A) Protein expression levels of ROCK1 and NFATc3 were measured by western blotting; corresponding histograms of (B) ROCK1 and (C) NFATc3 protein bands normalized to those of $\beta$-actin. The mRNA expression of (D) ROCK1, (E) NFATc3 and (F) miR-153 in HPASMCs exposed to hypoxic conditions. Data represent the mean \pm standard deviation, $\mathrm{n}=3$. Comparisons were performed using one-way ANOVA followed by Dunnett's post-hoc test. ${ }^{*} \mathrm{P}<0.05$ and ${ }^{* *} \mathrm{P}<0.01$ vs. control at day 0 . ROCK1, $\mathrm{Q}-\mathrm{ass}$. kinase 1; NFATc3, nuclear factor of activated T cells cytoplasmic 3; HPASMCs, human pulmonary artery smooth muscle cells; miR, microRNA.

Fisher Scientific, Inc.) was used to mutate the complementary binding site (CUAUGCA) in the ROCK1-WT vector to GUCUCCC. In order to establish the NFATc3 mutant luciferase reporter vector NFATc3 (-MUT), the mutated complementary binding site (AAUGUGA) located in the NFATc3-WT vector was mutated to CGGACUA. Then, $293 \mathrm{~T}$ cells were transfected with $100 \mathrm{ng}$ ROCK1-WT or NFATc3-WT or ROCK1-MUT or NFATc3-MUT vector and $500 \mathrm{ng}$ miR-153 mimic for $48 \mathrm{~h}$ at $37^{\circ} \mathrm{C}$. Luciferase activity was measured with a Lumat LB9508 luminometer (Berthold, Bad Wildbad, Germany). Relative luciferase intensity was normalized to Renilla luciferase activity.

Statistical analysis. The data collected were statistically evaluated and analyzed with SPSS v18.0 (SPSS, Inc.). All data are expressed as mean \pm standard deviation. Comparisons between the two unpaired groups were performed using an unpaired Student's t-test. Differences between multiple groups were tested by one-way analysis of variance, followed by a Dunnett's post-hoc test. $\mathrm{P}<0.05$ was considered to indicate a statistically significant difference.

\section{Results}

Effect of chronic hypoxia on RVSP, RVHI, WA\% and WT\% in rats. As shown in Fig. $1 \mathrm{~A}$ and $\mathrm{B}$, the RVSP and RVHI values were significantly increased from 2 and 4 weeks after the rats were exposed to hypoxia $(\mathrm{P}<0.01)$, respectively. The WA\% (Fig. 1C) and WT\% (Fig. 1D) values in rats under hypoxia were significantly elevated from 4 weeks onwards $(\mathrm{P}<0.05)$. After 6 weeks of hypoxia, the WT\% value in hypoxic rats was 1.9-fold higher than the level in normoxic control rats and the WA\% values were 1.7-fold higher compared with the level in the control group. Hematoxylin and eosin staining demonstrated marked thickening of the smooth muscle layer of pulmonary vessels in the hypoxic group, with narrowing of the lumen and inflammatory cell infiltration (Fig. 1E). Morphological investigation showed the proliferation and migration of PASMCs.
Upregulated expression levels of ROCK1 and NFATc3 proteins in hypoxic rats. At five time-points (0, 1, 2, 4 and 6 weeks) after exposure to hypoxia, six rats in each group were sacrificed and the pulmonary arteries removed. Western blotting was performed to confirm the expression levels of ROCK1 and NFATc3 proteins in pulmonary arteries; $\beta$-actin was used as the internal reference for ROCK1 and NFATc3. As shown in Fig. 2A, the expression levels of ROCK1 and NFATc3 proteins were increased from 2 and 4 weeks after exposure to hypoxia, respectively. After 6 weeks, the expression levels of ROCK1 and NFATc3 proteins were upregulated (2.1- and 2.3-fold, respectively) compared with the level in the control group at day 0 (Fig. 2B and C).

miR-153, ROCK1 and NFATc3 expressions are regulated by hypoxia in HPASMCs. From the results of rat models, the protein expression levels of ROCK1 and NFATc3 were significantly increased from 2 week after the rats were exposed to hypoxia $(\mathrm{P}<0.05)$. Given the rapid changes of ROCK1 and NFATc3 protein expressions under hypoxia, the gene changes within $24 \mathrm{~h}$ were determined using HPASMCs under hypoxic conditions. After exposure of HPASMCs to hypoxic conditions from 0 to $24 \mathrm{~h}$, mRNA expressions of ROCK1 and NFATc3 were clearly increased (Fig. 2D and E). At the same time, the mRNA expression of miR-153 was significantly decreased (Fig. 2F).

Validation of miR-153 mimic, mimic control, miR-153 inhibitor and inhibitor control transfection into HPASMCs. The immunofluorescence intensity of miR-153 in HPASMs was increased after transfection of miR-153 mimic compared with transfection with mimic control. RT-qPCR was used to detect the expression level of miR-153 in HPASMCs following transfection; the relative expression was normalized to that of U6, with that of inhibitor control set as 1. Compared with control cells, miR-153 expression was markedly increased in cells transfected with the miRNA-153 mimic and was decreased in cells transfected with the miRNA-153 inhibitor 
A
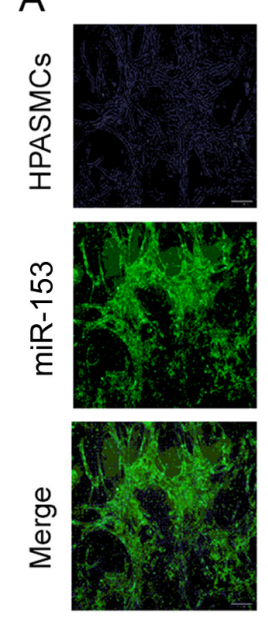
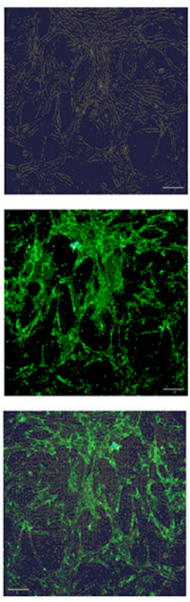
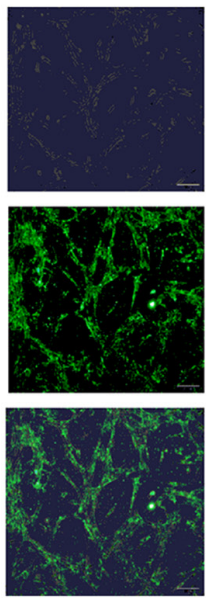
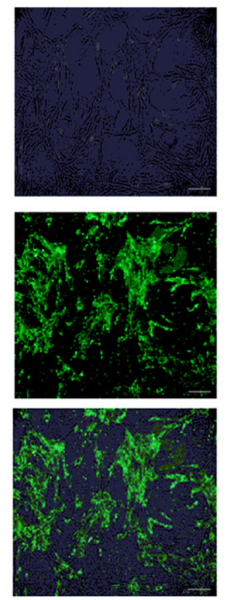

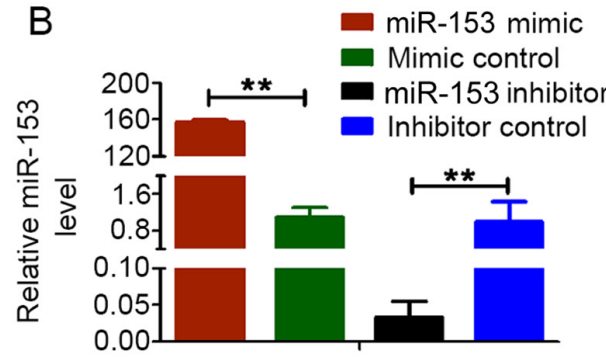

Figure 3. HPASMCs were transfected with lentivirus vectors expressing the miR-153 mimic, mimic control, miR-153 inhibitor and mimic control. The transfection efficiency was estimated by (A) evaluation of GFP expression under a fluorescence microscope and (B) analyzed by reverse transcription-quantitative PCR. The first line of images in (A) was acquired with a brightfield lens. Data represent the mean \pm standard deviation. $n=3$. " $P<0.01$ vs. inhibitor control group. HPASMCs, human pulmonary artery smooth muscle cells; miR, microRNA; GFP, green fluorescent protein.

A

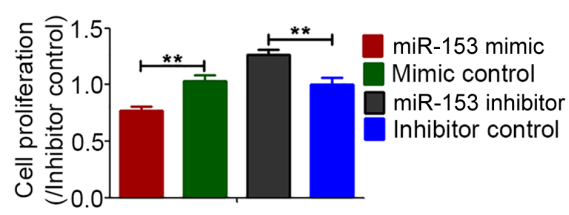

C

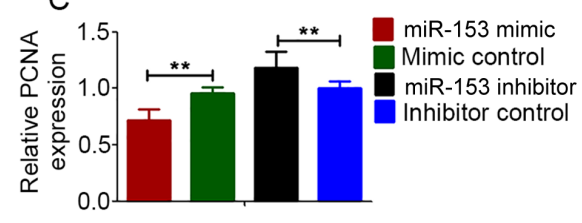

$\mathrm{E}$

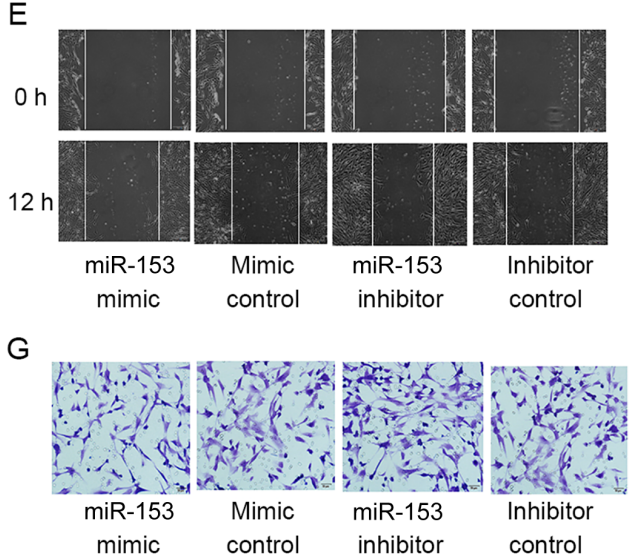

B

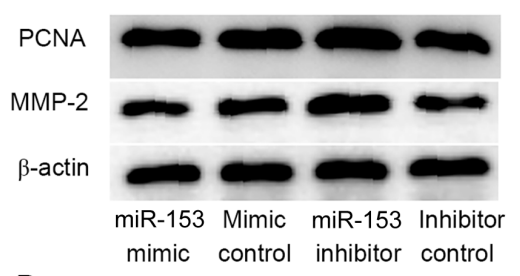

D
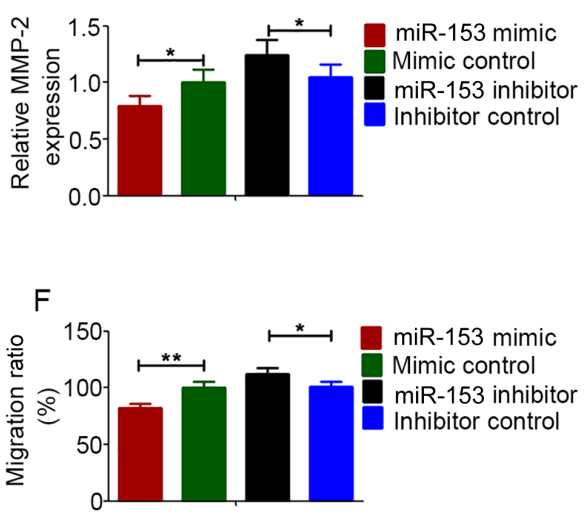

$\mathrm{H}$

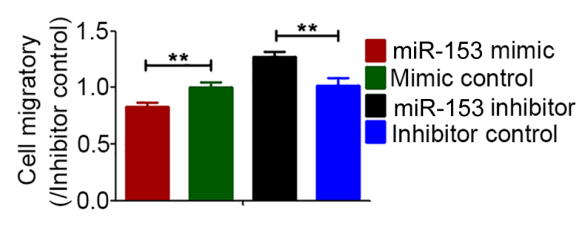

Figure 4. miR-153 inhibited hypoxia-induced proliferation and migration capacity of HPASMCs. (A) CCK-8 and (B-D) western blot assays analyzed the cell proliferation; (E and F) wound healing. Scale bar, $200 \mu \mathrm{m}$. (G and H) Transwell assays for cell migration. Scale bar, $50 \mu \mathrm{m}$. Data represent the mean \pm standard deviation. $\mathrm{n}=3 .{ }^{*} \mathrm{P}<0.05,{ }^{* *} \mathrm{P}<0.01$ vs. inhibitor control. miR, microRNA; HPASMCs, human pulmonary artery smooth muscle cells.

(Fig. 3). These observations demonstrated that miR-153 mimic, mimic control, miR-153 inhibitor and inhibitor control were successfully transfected into HPASMCs and could be used in subsequent experiments.
Effects of miR-153 on the proliferation and migration of HPASMCs under hypoxic conditions. The proliferation and migration of HPASMCs are considered to be the cause of pulmonary vascular remodeling induced by chronic hypoxia (28). 
A

SV40 Promoter
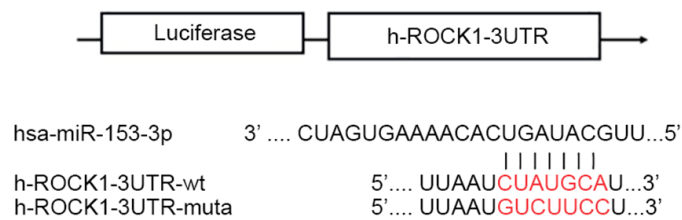

B

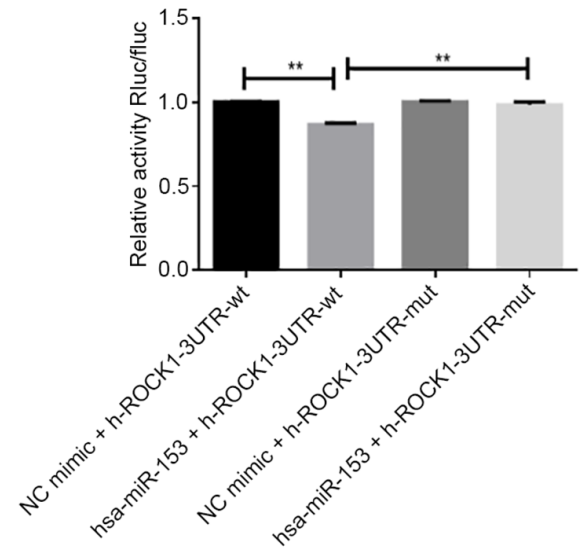

C

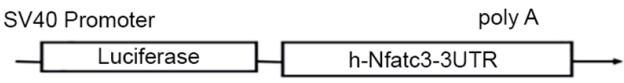

hsa-miR-153-3p 3'.... CUAGUGAAAACACUGAUACGUU ...5' h-Nfatc3-3UTR-wt1
h-Nfatc3-3UTR-muta1

hsa-miR-153-3p 3'... CUAGUGAAAACACUGAUACGUU...5'

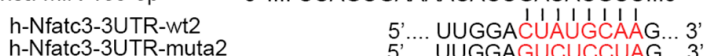

D

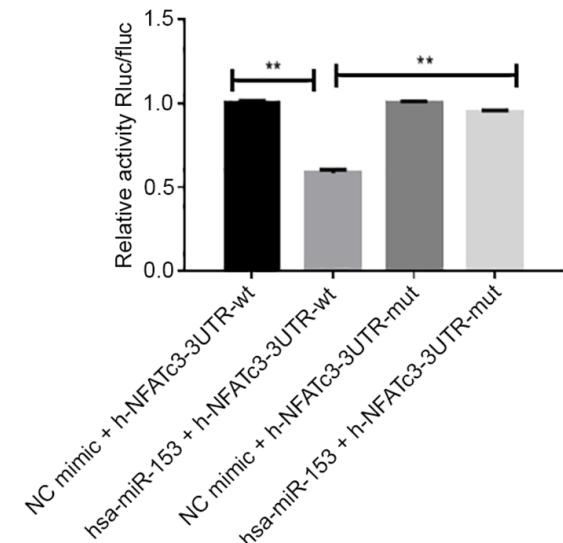

Figure 5. miR-153 directly targeted 3'-UTR of ROCK1 or NFATc3 in 293T cells. (A) One region in the ROCK1 3'-UTR was predicted to bind with miR-153. (B) Analysis of luciferase intensity in 293T cells co-transfected with mimic miR-153 or mimic NC and plasmid containing wt ROCK1 3'-UTR or mut ROCK1 3'-UTR. (C) Two regions in the NFATc3 3'-UTR were predicted to bind with miR-153. (D) Analysis of luciferase intensity in 293T cells co-transfected with mimic miR-153 or mimic NC and plasmid containing wild-type NFATc3-3'-UTR or mutant NFATc-3'-UTR. Data represent the mean \pm standard deviation. $\mathrm{n}=3$. ${ }^{* *} \mathrm{P}<0.01$. miR, microRNA; 3'-UTR, 3'-untranslated region; ROCK1, Q-associated, coiled-coil-containing protein kinase 1; NFATc3, nuclear factor of activated T cells cytoplasmic 3; NC, negative control; wt, wild-type; mut, mutant.

A

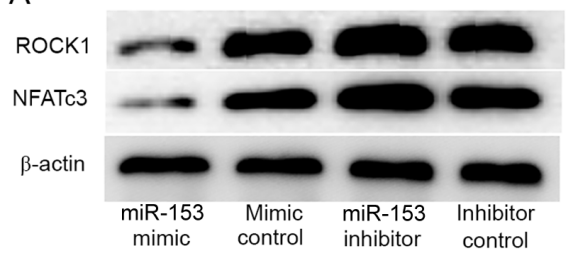

B

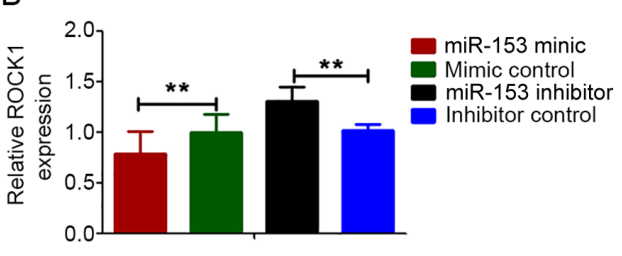

C

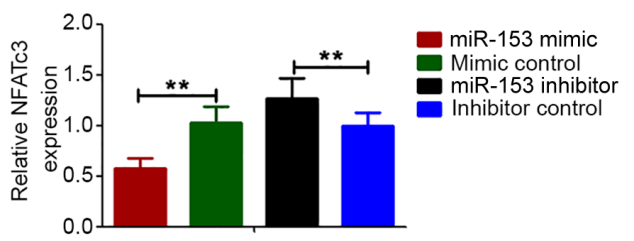

D
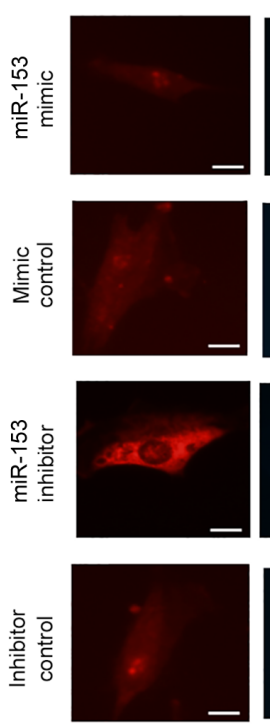

DAPI
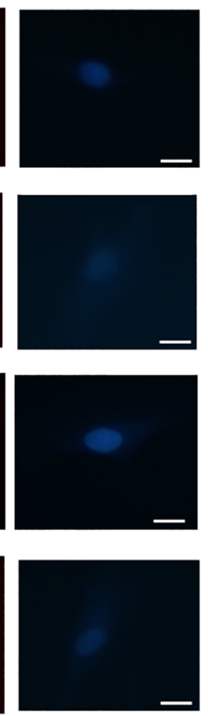

Merge
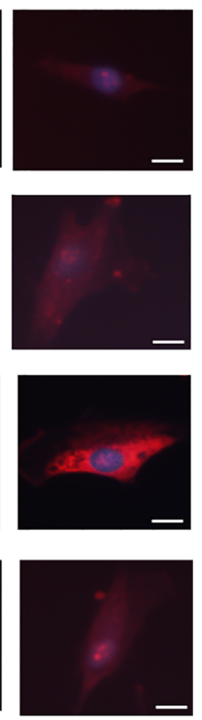

Figure 6. miR-153 inhibited the expression of ROCK1 and NFATc3 proteins in HPASMCs. (A) Western blotting showing the protein expression levels of ROCK1 and NFATc3. Quantification of the western blotting analysis for the protein expression of (B) ROCK1 and (C) NFATc3. $\beta$-actin was used as the internal control and the expression value of inhibitor control was set to 1 . Data represent the mean \pm standard deviation. $n=3$. ${ }^{* *} \mathrm{P}<0.01$. (D) miR-153 mimics attenuated the expression of NFATc3 proteins in HPASMCs and the miR-153 inhibitor promoted the expression of NFATc3 proteins in HPASMCs. (scale bar=100 $\mu$ m). miR, microRNA; ROCK1, @-associated, coiled-coil-containing protein kinase 1; NFATc3, nuclear factor of activated T cells cytoplasmic 3; HPASMCs, human pulmonary artery smooth muscle cells.

To determine the regulatory effect of miR-153 on HPASMCs under hypoxic conditions, miR-153 was overexpressed in HPASMCs and then cell proliferation and migration were analyzed under anoxic conditions. Cell proliferation was determined using CCK-8 assays. MMP-2 and PCNA expression was measured by western blot analysis. All the results demonstrated 
that overexpression of miR-153 inhibited the proliferation of HPASMCs under hypoxic conditions, while the miR-153 inhibitor enhanced the proliferation of HPASMCs (Fig. 4A-C). Cell migration abilities were determined using wound healing and Transwell assays. Under hypoxic conditions, overexpression of miR-153 significantly inhibited the migration abilities of HPASMCs, while the miR-153 inhibitor enhanced the migration abilities of HPASMCs (Fig. 4B and D-H).

miR-153 directly targets ROCK1 or NFATc 3 in 2937 cells. To further explore the molecular mechanism by which miR-153 regulated the proliferation and migration of HPASMCs under hypoxic conditions, TargetScan and miRanda were used to predict the potential target sites of miR-153 in ROCK1 or NFATc 3 mRNA. A region in the ROCK1 mRNA was predicted to be a miR-153 binding site (Fig. 5A) and two regions in the NFATc3 mRNA were predicted to be miR-153 ROCK1 3'-UTR or NFATc3-3'-UTR mutant plasmids (Fig. 5C).

Dual luciferase assays were then performed to confirm the binding association between miR-153 and ROCK1 or NFATc3. As shown in Fig. 5B and D, the luciferase activity of the reporter was inhibited in 293T cells co-transfected with miR-153 mimic compared with those co-transfected the mimic control. However, there was no significant change in the fluorescence intensity after mutation of the binding site, suggesting that miR-153 interacted directly with ROCK1 or NFATc3 at this binding site.

Effect of miR-153 on the expression of ROCK1 and NFATc3 proteins in HPASMCs under hypoxic conditions. It has been reported that ROCK1 and NFATc3 expressions are related to PAH (29,30). The present study investigated whether miR-153 regulated the proliferation and migration of HPASMCs induced by hypoxia by targeting ROCK1 and NFATc3. HPASMCs were transfected with lentivirus vectors expressing the miRNA-153 mimic, miRNA-153 inhibitor and non-specific control and then they were treated with hypoxia for $12 \mathrm{~h}$. Compared with the control cells, the expression levels of ROCK1 and NFATc3 proteins were markedly decreased in cells transfected with the miRNA-153 mimic and were increased in cells transfected with the miRNA-153 inhibitor (Fig. 6A-C). This result was consistent with immunofluorescence experiment in that the expression of NFATc3 increased after inhibition of miRNA-153, while the expression of NFATc3 decreases after overexpression of miRNA-153 (Fig. 6D).

\section{Discussion}

Chronic hypoxia is known to cause pulmonary hypertension (31). The major pathological changes associated with hypoxia-induced pulmonary hypertension (HPH) are pulmonary vasoconstriction and remodeling resulting from PAMSCs proliferation (32). The present study successfully established a model of HPH. The RVSP, RVHI, WA\% and WT\% values of rats in the model group exposed to hypoxia were significantly increased compared with those of normal rats, suggesting that hypoxia causes RV failure and pulmonary vascular remodeling. Electron microscopic evaluation of the pulmonary vascular structure revealed abnormal proliferation and migration of PAMSCs. In addition, hypoxia significantly increased the thickness of lung vessels and the proportion of muscularized pulmonary vessels. In vitro studies were performed to confirm the effects of hypoxia on PAMSCs.

ROCK1 and NFATc3 are involved in the regulation of pulmonary vascular remodeling induced by hypoxia $(30,33)$. In the present study, expressions of ROCK1 and NFATc3 proteins in the pulmonary vasculature were determined after rats were exposed to hypoxia for $0,1,2,4$ and 6 weeks. The results demonstrated significantly increased expression of ROCK1 and NFATc3 proteins during the period of exposure to hypoxia. ROCK1 and NFATc3 mRNA levels were evaluated after HPASMCs were cultured under hypoxia for 0, 6, 12 and $24 \mathrm{~h}$ and ROCK 1 and NFATc 3 expressions were significantly upregulated. Given the important roles of ROCK1 and NFATc3 in the formation of HPH $(34,35)$, ROCK1 and NFATc3 were related to the pathological process of HPH and may even participate in this process.

Recently, a series of studies demonstrated that hypoxic modulation of cellular function could be mediated by miRNAs during the development of cancer (36-38). HPH and cancer have similar phenotypes because both diseases are characterized by increased cell proliferation and migration $(39,40)$. The present study chose miR-153 as the research target because previous studies have reported its antitumor function in different types of cancer including papillary thyroid and breast cancer $(41,42)$. However, little is known about its expression and function in HPASMCs in hypoxia. The present study revealed that miR-153 expression decreased in hypoxia-treated HPASMCs. It was also found that miR-153 inhibitor increased HPASMC proliferation and migration, while the miR-153 mimic reversed the increased proliferation and migration of HPASMCs induced by hypoxia, which is consistent with previous studies in cancer cells (43-45). All these data suggested that miR-153 regulated hypoxia-induced cell proliferation and migration.

The above data confirmed that miR-153 exhibited preventive potential in the proliferation and migration of HPASMCs induced by hypoxia, although the targets of miR-153 remained elusive. ROCK1 and NFATc 3 are important therapeutic targets for PAH and they are activated and involved in the modulation of cell proliferation and migration in PAH $(46,47)$. Therefore, an in-depth analysis was conducted using TargetScan to determine whether NFATc3 and ROCK1 are the target genes of miR-153. The dual-luciferase experiments verified that miR-153 directly targeted NFATc3 and ROCK1. Using $293 \mathrm{~T}$ cells, it was confirmed that miR-153 interacted with the 3'-UTR of ROCK1 and NFATc3. Western blotting and immunofluorescence analysis demonstrated that miR-153 significantly decreased the protein expressions of ROCK1 and NFATc3. Thus, it was hypothesized that miR-153 directly targets ROCK1 and NFATc3 to inhibit HPH. The translocation of dephosphorylated NFATc3 from the cytoplasm to the nucleus is essential for the subsequent activation of its target genes (24). Future studies will investigate the effect of miR-153 on nuclear translocation of NFATc3. In addition, an HPH model of rats will be used to explore the miR-153 expression in hypoxic pulmonary arteries with different exposure time and confirm the effect of miR-153 on HPH.

In summary, the present study is the first, to the best of the authors' knowledge, to provide evidence that miR-153 inhibited hypoxia-induced proliferation and migration of HPASMCs, 
at least partly, through targeting ROCK1 and NFATc3. The present study highlighted the biological significance of the miR-153/ROCK1 and miR-153/NFATc3 axes in HPASMCs under hypoxia. Further in vivo studies should be performed to confirm that miR-153 is a promising candidate for PAH therapy.

\section{Acknowledgements}

Not applicable.

\section{Funding}

The present study was supported by the National Nature Science Foundation of China (grant no. 81700050).

\section{Availability of data and materials}

All data generated or analyzed during the present study are included in this published article.

\section{Authors' contributions}

MZ, LS and WW participated in the design of the paper and experiment. MZ, YL and LS participated in the preliminary experiment and the processing of experimental results. DK and NW prepared the figures and conducted data analysis. MZ and YL were involved in drafting the manuscript. All authors read and approved the final manuscript.

\section{Ethics approval and consent to participate}

The present study adhered to the Guide for the Care and Use of Laboratory Animals published by the US National Institutes of Health (NIH Publication No. 85-23, revised 1996) and all experimental protocols were approved by the Animal Experimentation Ethics Committee of Jinzhou Medical University (approval no. 2019065).

\section{Patient consent for publication}

Not applicable.

\section{Competing interests}

The authors declare that they have no competing interests.

\section{References}

1. Kahraman BO, Savci S, Ozsoy I, Baran A, Acar S, Ozpelit E, Balci A, Sevinc C and Akdeniz B: Effects of neuromuscular electrical stimulation in patients with pulmonary arterial hypertension: A randomized controlled pilot study. J Cardiol 75: 702-708, 2020.

2. Hiraide T, Kataoka M, Suzuki H, Aimi Y, Chiba T, Isobe S, Katsumata Y, Goto S, Kanekura K, Yamada Y, et al: Poor outcomes in carriers of the RNF213 variant (p.Arg4810Lys) with pulmonary arterial hypertension. J Heart Lung Transplant 39: 103-112, 2020.

3. Zhang L, Wang Y, Wu G, Rao L, Wei Y, Yue H, Yuan T, Yang P, Xiong F, Zhang S, et al: Blockade of JAK2 protects mice against hypoxia-induced pulmonary arterial hypertension by repressing pulmonary arterial smooth muscle cell proliferation. Cell Prolif 53: $\mathrm{e} 12742,2020$

4. Luo F, Wang X, Luo X, Li B, Zhu D, Sun H and Tang Y: Invasive hemodynamic assessment for the right ventricular system and hypoxia-induced pulmonary arterial hypertension in mice. J Vis Exp 152: e60090, 2019.
5. Zhou W, Negash S, Liu J and Raj JU: Modulation of pulmonary vascular smooth muscle cell phenotype in hypoxia: Role of cGMP-dependent protein kinase and myocardin. Am J Physiol Lung Cell Mol Physiol 296: L780-L789, 2009.

6. Humbert M, Sitbon O, Chaouat A, Bertocchi M, Habib G, Gressin V, Yaïci A, Weitzenblum E, Cordier JF, Chabot F, et al: Survival in patients with idiopathic, familial, and anorexigen-associated pulmonary arterial hypertension in the modern management era. Circulation 122: 156-163, 2010.

7. Ren ZP, Hou XB, Tian XD, Guo JT, Zhang LB, Xue ZQ, Deng JQ, Zhang SW, Pan JY and Chu XY: Identification of nine microRNAs as potential biomarkers for lung adenocarcinoma. FEBS Open Bio 9: 315-327, 2019.

8. Peng XX, Yu R, Wu X, Wu SY, Pi C, Chen ZH, Zhang XC, Gao CY, Shao YW, Liu L, et al: Correlation of plasma exosomal microRNAs with the efficacy of immunotherapy in EGFR/ALK wild-type advanced non-small cell lung cancer. J Immunother Cancer 8: e000376, 2020. Erratum in: J Immunother Cancer 8: e000376corr1, 2020.

9. Kim D, Kim Y and Kim Y: Effects of $\beta$-carotene on expression of selected micrornas, histone acetylation, and DNA methylation in colon cancer stem cells. J Cancer Prev 24: 224-232, 2019.

10. Moloney BM, Gilligan KE, Joyce DP, O'Neill CP, O'Brien KP, Khan S, Glynn CL, Waldron RM, Maguire CM, Holian E, et al: Investigating the potential and pitfalls of EV-encapsulated microRNAs as circulating biomarkers of breast cancer. Cells 9: 141,2020

11. Zhao L, Shan Y, Liu B, Li Y and Jia L: Functional screen analysis reveals miR-3142 as central regulator in chemoresistance and proliferation through activation of the PTEN-AKT pathway in CML. Cell Death Dis 8: e2830, 2017. Retraction in: Cell Death Dis 11: 121,2020

12. Zhu TT, Zhang WF, Yin YL, Liu YH, Song P, Xu J, Zhang MX and Li P: MicroRNA-140-5p targeting tumor necrosis factor- $\alpha$ prevents pulmonary arterial hypertension. J Cell Physiol 234: 9535-9550, 2019.

13. Babicheva A, Ayon RJ, Zhao T, Ek Vitorin JF, Pohl NM, Yamamura A, Yamamura H, Quinton BA, Ba M, Wu L, et al: MicroRNA-mediated downregulation of $\mathrm{K}^{+}$channels in pulmonary arterial hypertension. Am J Physiol Lung Cell Mol Physiol 318: L10-L26, 2020.

14. Mohsenin V:The emerging role of microRNAsin hypoxia-induced pulmonary hypertension. Sleep Breath 20: 1059-1067, 2016.

15. Wang P, Xu J, Hou Z, Wang F, Song Y, Wang J, Zhu H and Jin H: miRNA-34a promotes proliferation of human pulmonary artery smooth muscle cells by targeting PDGFRA. Cell Prolif 49: 484-493, 2016.

16. Tang BI, Tang MM, Xu QM, Guo JL, Xuan L, Zhou J, Wang XJ, Zhang H and Kang PF: MicroRNA-143-5p modulates pulmonary artery smooth muscle cells functions in hypoxic pulmonary hypertension through targeting HIF-1 $\alpha$. J Biosci 45: 37, 2020.

17. Shan N, Shen L, Wang J, He D and Duan C: MiR-153 inhibits migration and invasion of human non-small-cell lung cancer by targeting ADAM19. Biochem Biophys Res Commun 456: 385-391, 2015.

18. Wang L, Lv X, Fu X, Su L, Yang T and Xu P: MiR-153 inhibits the resistance of lung cancer to gefitinib via modulating expression of ABCE1. Cancer Biomark 25: 361-369, 2019.

19. Liang C, Li X, Zhang L, Cui D, Quan X and Yang W: The anti-fibrotic effects of microRNA-153 by targeting TGFBR-2 in pulmonary fibrosis. Exp Mol Pathol 99: 279-285, 2015.

20. Liang H, Xiao J, Zhou Z, Wu J, Ge F, Li Z, Zhang H, Sun J, Li F, Liu R, et al: Hypoxia induces miR-153 through the IRE1 $\alpha-X B P 1$ pathway to fine tune the HIF1 $\alpha$ /VEGFA axis in breast cancer angiogenesis. Oncogene 37: 1961-1975, 2018.

21. Liu WH, Xu XH, Luo Q, Zhang HL, Wang Y, Xi QY, Zhao ZH and Liu ZH: Inhibition of the RhoA/Rho-associated, coiled-coil-containing protein kinase-1 pathway is involved in the therapeutic effects of simvastatin on pulmonary arterial hypertension. Clin Exp Hypertens 40: 224-230, 2018.

22. Zhang M, Chang Z, Zhang P, Jing Z, Yan L, Feng J, Hu Z, Xu Q, Zhou W, Ma P, et al: Protective effects of $18 \beta$-glycyrrhetinic acid on pulmonary arterial hypertension via regulation of Rho A/Rho kinsase pathway. Chem Biol Interact 311: 108749, 2019.

23. Zhu J, Liu C, Wang B, Tong X and Li Z: Qibai Pingfei capsule medicated serum inhibits the proliferation of hypoxia-induced pulmonary arterial smooth muscle cells via the $\mathrm{Ca}^{2+} /$ calcineurin/nuclear factor of activated T-cells 3 pathway. J Tradit Chin Med 37: 466-474, 2017. 
24. Kang K, Peng X, Zhang X, Wang Y, Zhang L, Gao L, Weng T, Zhang H, Ramchandran R, Raj JU, et al: MicroRNA-124 suppresses the transactivation of nuclear factor of activated $\mathrm{T}$ cells by targeting multiple genes and inhibits the proliferation of pulmonary artery smooth muscle cells. J Biol Chem 288 25414-25427, 2013.

25. Hasnat AK, van der Velde ET, Hon JKF and Yacoub MH: Reproducible model of post-infarction left ventricular dysfunction: haemodynamic characterization by conductance catheter. Eur J Cardiothorac Surg 24: 98-104, 2003.

26. Livak KJ and Schmittgen TD: Analysis of relative gene expression data using real-time quantitative PCR and the $2-\Delta \Delta C$ T method. Methods 25: 402-408, 2001.

27. Agarwal V, Bell GW, Nam JW and Bartel DP: Predicting effective microRNA target sites in mammalian mRNAs. Elife 4: $\mathrm{e} 05005,2015$.

28. Zhao M, Chen N, Li X, Lin L and Chen X: miR-19a modulates hypoxia-mediated cell proliferation and migration via repressing PTEN in human pulmonary arterial smooth muscle. Life Sci 239: $116928,2019$.

29. Siddique MAH, Satoh K, Kurosawa R, Kikuchi N, Elias-Al-Mamun M, Omura J, Satoh T, Nogi M, Sunamura S, Miyata S, et al: Identification of emetine as a therapeutic agent for pulmonary arterial hypertension: novel effects of an old drug. Arterioscler Thromb Vasc Biol 39: 2367-2385, 2019.

30. Han J, Tian H, Liu Y and Fan F: Sarpogrelate attenuates pulmonary arterial hypertension via calcium/calcineurin axis. Front Biosci 24: 607-615, 2019.

31. Yu L, Quinn DA, Garg HG and Hales CA: Deficiency of the NHE1 gene prevents hypoxia-induced pulmonary hypertension and vascular remodeling. Am J Respir Crit Care Med 177: 1276-1284, 2008.

32. Wu J, Pan W, Wang C, Dong H, Xing L, Hou J, Fang S, Li H, Yang $\mathrm{F}$ and $\mathrm{Yu} \mathrm{B}: \mathrm{H}_{2} \mathrm{~S}$ attenuates endoplasmic reticulum stress in hypoxia-induced pulmonary artery hypertension. Biosci Rep 39: BSR20190304, 2019

33. Wu W, Li Y and Xu DQ: Role of ROS/Kv/HIF Axis in the development of hypoxia-induced pulmonary hypertension. Chin Med Sci J 32: 253-259, 2017

34. Wang Y, Duo D, Yan Y, He R and Wu X: Magnesium lithospermate B ameliorates hypobaric hypoxia-induced pulmonary arterial hypertension by inhibiting endothelial-to-mesenchymal transition and its potential targets. Biomed Pharmacother 130 110560,2020

35. Bierer R, Nitta $\mathrm{CH}$, Friedman J, Codianni S, de Frutos $\mathrm{S}$, Dominguez-Bautista JA, Howard TA, Resta TC and Bosc LV: NFATc3 is required for chronic hypoxia-induced pulmonary hypertension in adult and neonatal mice. Am J Physiol Lung Cell Mol Physiol 301: L872-L880, 2011.

36. Shao H, Dong D and Shao F: Long non-coding RNA TUG1-mediated down-regulation of KLF4 contributes to metastasis and the epithelial-to-mesenchymal transition of colorectal cancer by miR-153-1. Cancer Manag Res 11: 8699-8710, 2019
37. Xiao L, He Y, Peng F, Yang J and Yuan C: Endometrial cancer cells promote M2-like macrophage polarization by delivering exosomal miRNA-21 under hypoxia condition. J Immunol Res 2020: 9731049, 2020.

38. Yang Y, Qu A, Wu Q, Zhang X, Wang L, Li C, Dong Z, Du L and Wang C: Prognostic value of a hypoxia-related microRNA signature in patients with colorectal cancer. Aging (Albany NY) 12: 35-52, 2020.

39. Miao R, Liu W, Qi C, Song Y, Zhang Y, Fu Y, Liu W, Lang Y, Zhang Y and Zhang Z: miR-18a-5p contributes to enhanced proliferation and migration of PASMCs via targeting Notch2 in pulmonary arterial hypertension. Life Sci 257: 117919, 2020.

40. Peng $\mathrm{Y}$ and Wang HH: Cir-ITCH inhibits gastric cancer migration, invasion and proliferation by regulating the Wnt/ $\beta$-catenin pathway. Sci Rep 10: 17443, 2020

41. Luo Y, Teng X, Zhang L, Chen J, Liu Z, Chen X, Zhao S, Yang S, Feng J and Yan X: CD146-HIF-1 $\alpha$ hypoxic reprogramming drives vascular remodeling and pulmonary arterial hypertension. Nat Commun 10: 3551, 2019. Erratum in: Nat Commun 10: 4098 , 2019.

42. Cui Z, Luo Z, Lin Z, Shi L, Hong Y and Yan C: Long non-coding RNA TTN-AS1 facilitates tumorigenesis of papillary thyroid cancer through modulating the miR-153-3p/ZNRF2 axis. J Gene Med 21: e3083, 2019.

43. Zuo Z, Ye F, Liu Z, Huang J and Gong Y: MicroRNA-153 inhibits cell proliferation, migration, invasion and epithelial-mesenchymal transition in breast cancer via direct targeting of RUNX2. Exp Ther Med 17: 4693-4702, 2019.

44. Wang J, Liang S and Duan X: Molecular mechanism of miR-153 inhibiting migration, invasion and epithelial-mesenchymal transition of breast cancer by regulating transforming growth factor beta (TGF- $\beta$ ) signaling pathway. J Cell Biochem 120: 9539-9546, 2019.

45. Ma H, Tian T, Liu X, Xia M, Chen C, Mai L, Xie S and Yu L: Upregulated circ_0005576 facilitates cervical cancer progression via the miR-153/KIF20A axis. Biomed Pharmacother 118: 109311, 2019.

46. He RL, Wu ZJ, Liu XR, Gui LX, Wang RX and Lin MJ: Calcineurin/NFAT signaling modulates pulmonary artery smooth muscle cell proliferation, migration and apoptosis in monocrotaline-induced pulmonary arterial hypertension rats. Cell Physiol Biochem 49: 172-189, 2018.

47. Huetsch JC, Walker J, Undem C, Lade J, Yun X, Baksh S, Jiang H, Lai $\mathrm{N}$ and Shimoda LA: Rho kinase and $\mathrm{Na}^{+} / \mathrm{H}^{+}$exchanger mediate endothelin-1-induced pulmonary arterial smooth muscle cell proliferation and migration. Physiol Rep 6: e13698, 2018.

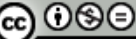

This work is licensed under a Creative Commons Attribution-NonCommercial-NoDerivatives 4.0 International (CC BY-NC-ND 4.0) License. 